\title{
Spleen tyrosine kinase regulates mammary epithelial cell proliferation in mammary glands of dairy cows
}

\author{
Xiaoming Hou, ${ }^{*}$ Lin Lin, ${ }^{*}$ Weinan Xing, ${ }^{*}$ Yang Yang, ${ }^{*}$ Xiaoyu Duan, ${ }^{*}$ Qingzhang Li, ${ }^{*} \dagger$ Xuejun Gao, ${ }^{*} \dagger$ \\ and Ye Lin* ${ }^{1}$ \\ ${ }^{*}$ College of Life Science, and \\ †Key Laboratory of Dairy Science of Education Ministry, Northeast Agricultural University, Harbin 150030, China
}

\begin{abstract}
Spleen tyrosine kinase (SYK) is a nonreceptor tyrosine kinase that has been considered a hematopoietic cell-specific signal transducer involved in cell proliferation and differentiation. However, the role of SYK in normal mammary gland is still poorly understood. Here we show that SYK is expressed in mammary glands of dairy cows. Expression of SYK was higher in dry period mammary tissues than in lactating mammary tissues. Knockdown and overexpression of SYK affected dairy cow mammary epithelial cell proliferation as well as the expression of signal molecules involved in proliferation, including protein kinase $\mathrm{B}$ ( $\mathrm{PKB}$, also known as AKT1), p42/44 mitogen-activated protein kinase (MAPK), and signal transducer and activator of transcription 5 (STAT5). Dual-luciferase reporter assay showed that SYK increased the transcriptional activity of the AKT1 promoter, and cis-elements within the AKT1 promoter region from -439 to $-84 \mathrm{bp}$ mediated this regulation. These results suggest that SYK affects mammary epithelial cell proliferation by activating $A K T 1$ at the transcriptional level in mammary glands of dairy cows, which is important for the mammary remodeling process in dry cows as well as for increasing persistency of lactation in lactating cows.
\end{abstract}

Key words: mammary gland, SYK, proliferation, AKT1

\section{INTRODUCTION}

Bovine milk is an agricultural product with tremendous economic importance worldwide because it is the primary source of nutrition for growth and development

Received July 15, 2015.

Accepted January 17, 2016.

${ }^{1}$ Corresponding author: linlu516@163.com of the calf (Nissen et al., 2013), as well as an important part of human nutrition (Haug et al., 2007). Bovine milk is synthesized in the mammary epithelial cells of dairy cows (Ollier et al., 2007). Many physiological factors, such as nutrition and pregnancy status, and management factors, such as milking frequency and efficiency, influence milk yield and composition (Stefanon et al., 2002; Nørgaard et al., 2008a; Dessauge et al., 2011). At the cell level, studies on mammary cell loss and replacement during lactation indicate that the number of mammary secretory cells and the secretory activity per cell determine milk yield (Capuco et al., 2003). The number of mammary epithelial cells is greatest during early lactation and declines with advancing lactation (Capuco et al., 2001). Thus, it is important to maintain high cell number by either increasing mammary cell survival and proliferation or reducing apoptosis during lactation.

Following cessation of milking or weaning of the young, a dry period occurs. The dry period is necessary to facilitate cell turnover in the bovine mammary gland and to optimize milk production in the next lactation cycle (Capuco et al., 1997). A typical dry period in a dairy cow involves active involution after cessation of milk removal followed by a period of redevelopment before the next lactation cycle (Hurley, 1989). During the first days of active involution, extensive histological changes affect the capability of synthesis and secretion of milk components and a loss of integrity of the mammary epithelial cells are observed (Seeth et al., 2015). Thus, apoptosis is high early in the dry period and then cell proliferation increases (Nørgaard et al., 2008b).

The molecular mechanisms affecting mammary gland lactation cycle and milk quality are numerous, and they include gene expression, micro-RNA, and epigenetic regulation (Singh et al., 2012; Wang et al., 2012). Spleen tyrosine kinase (SYK) is a nonreceptor tyrosine kinase that is most highly expressed in hematopoietic cells and has the ability to regulate development and 
proliferation (Uckun et al., 2010). Upon receptor stimulation, a phospho-ITAM/(SYK-(SH2) $)_{2}$ interaction induces an allosteric activation of SYK by changing the conformation of SYK from a closed inactive structure to an open active form (Kulathu et al., 2009). Expression of SYK has been detected in normal mouse and human mammary tissues (Flück et al., 1995; Ruschel and Ullrich, 2004). The expression of SYK in the mammary gland promotes research into its potential role in lactation cycle and milk production. Studies in an IL-2-dependent natural killer cell line have shown that SYK is a critical effector essential for IL-2-mediated survival signaling upstream of protein kinase B (PKB, also known as AKT1; Jiang et al., 2003). In a murine hematopoietic cell line, expression of cytoplasmic TELSYK fusion protein leads to the constitutive activation of phosphoinositide 3-kinase (PI3K)/AKT, mitogenactivated protein kinase (MAPK) and signal transducer and activator of transcription 5 (STAT5), which are closely involved in cell proliferation (Kanie et al., 2004). However, a study directly comparing the expression of SYK in lactation cycle or in lactating cows with different milk quality has not been reported. Whether SYK affected lactation cycle and milk quality by inducing mammary epithelial cell proliferation has not been well established.

As SYK has the ability to induce cell proliferation, we hypothesized SYK may participate in mammary epithelial cell turnover in lactation cycle of dairy cows. Additionally, SYK may have a relationship with milk quality in lactating cows. To meet these objectives, we compared SYK expression in mammary glands of dry cows versus lactating cows and detected the effect of SYK on mammary epithelial cell proliferation. The expression and activity of proteins known to be involved in pathways regulating cell proliferation were examined by Western blot when SYK was knocked down or overexpressed. To evaluate if SYK was related to milk quality in lactating cows, we compared SYK expression in mammary glands of lactating cows with highquality milk (milk protein $>3 \%$ and milk fat $>3.5 \%$ ) versus low-quality milk (milk protein $<3 \%$ and milk fat $<3.5 \%$ ).

\section{MATERIALS AND METHODS}

\section{Animals and Tissue Collection}

In our study, all animal experimental protocols were approved by Northeast Agricultural University (Harbin, China). Nine multiparous Holstein cows with the similar genetic background were used to obtain mammary tissues: 6 were in the lactating period and 3 were
Table 1. Milk components of lactating Holstein dairy cows

\begin{tabular}{lcc}
\hline Milk component & $\begin{array}{c}\text { Dairy cows with } \\
\text { high-quality milk }\end{array}$ & $\begin{array}{c}\text { Dairy cows with } \\
\text { low-quality milk }\end{array}$ \\
\hline Milk protein (\%) & $3.27 \pm 0.04$ & $2.89 \pm 0.02$ \\
Milk fat (\%) & $4.17 \pm 0.01$ & $3.20 \pm 0.06$ \\
Lactose (\%) & $4.84 \pm 0.03$ & $4.52 \pm 0.09$ \\
DM (\%) & $11.93 \pm 0.01$ & $10.89 \pm 0.03$ \\
\hline
\end{tabular}

in the dry period. All animals were clinically healthy. All 6 lactating cows were in the third parity (calving at 52 to 54 mo of age) and at 90 DIM. Lactating cows were milked at 0800 and $1530 \mathrm{~h}$ daily. Three dry cows were pregnant and dried off at 310 DIM. Animals were housed in tiestalls and fed a ration based on grass silage and concentrate (Supplementary Table S1; http:// dx.doi.org/10.3168/jds.2015-10118). The feeding regimen was ad libitum throughout lactation and restricted during the dry period according to their requirements. Animals had free access to fresh water. Lactating cows were allocated to high-quality milk group (milk protein $>3 \%$ and milk fat $>3.5 \%, \mathrm{n}=3$ ) and low-quality milk group (milk protein $<3 \%$ and milk fat $<3.5 \%, \mathrm{n}=3$ ) according to their milk protein and fat contents (Table 1). Milk yield of lactating cows with high-quality milk was $33.9 \pm 2.1 \mathrm{~kg} / \mathrm{d}$. Milk yield of lactating cows with low-quality milk was $33.7 \pm 0.5 \mathrm{~kg} / \mathrm{d}$. Somatic cell count was $<50,000$ cells $/ \mathrm{mL}$ for all cows.

The 6 lactating cows were slaughtered at 90 DIM and the 3 dry cows were slaughtered at $30 \mathrm{~d}$ after dry off. The lactating cows were milked $1 \mathrm{~h}$ before slaughter. Immediately after exsanguination, several pieces of mammary parenchyma tissue were aseptically removed from the midregion of the mammary glands. Tissue samples were trimmed of visible connective and adipose tissues. Small pieces of mammary tissue were frozen in liquid nitrogen and stored at $-80^{\circ} \mathrm{C}$ for RNA and protein extraction or were fixed for immunofluorescence study. For cell culture, fresh tissue was placed in sterilized tubes containing ice-cold Hanks' balanced salt solution (HBSS, Life Technologies, Carlsbad, CA) and immediately transported to the laboratory.

\section{Reagents and Antibodies}

Dulbecco's Modified Eagle Medium/Nutrient Mixture F-12 (DMEM/F12), fetal bovine serum (FBS), TRIzol reagent, M-MLV, and Lipofectamine 2000 transfection reagent were purchased from Life Technologies. The RIPA buffer (P0013C) and mammalian genomic DNA extraction kit were purchased from Beyotime Biotechnology (Shanghai, China). The bicinchoninic acid (BCA) protein assay kit was purchased from Thermo 
Fisher Scientific Inc. (Waltham, MA). KeyFluor488 Click-iT 5-ethynyl-2'-deoxyuridine (EdU) Imaging Kit was purchased from KeyGEN BioTECH (Nanjing, China). The SYK, cyclin D1, STAT5, phospho-STAT5, and AKT1 antibodies were purchased from Santa Cruz Biotechnology Inc. (Santa Cruz, CA). Phospho-AKT1 antibody was purchased from Abcam (Cambridge, MA). The p42/44 MAPK, phospho-p42/44 MAPK, and $\beta$-actin antibodies, and SignalFire ECL reagent were purchased from Cell Signaling Technology (Beverly, MA). $\beta$-Casein antibody was purchased from Biosynthesis Biotechnology Co. Ltd. (Beijing, China). Horseradish peroxidase (HRP)-conjugated AffiniPure goat anti-rabbit IgG, HRP-conjugated AffiniPure rabbit anti-goat IgG, fluorescein isothiocyanate (FITC)conjugated AffiniPure goat anti-rabbit IgG, and goat serum were purchased from ZSGB-BIO (Beijing, China). The dual-luciferase reporter assay system was purchased from Promega Corporation (Madison, WI). Other reagents were purchased from Sigma-Aldrich (St Louis, MO) unless otherwise stated.

\section{Immunofluorescence Staining}

Mammary tissues were collected for immunofluorescence staining as described (Lin and Li, 2007). Briefly, $8-\mu \mathrm{m}$ thick cryosections were fixed in cold acetone for $10 \mathrm{~min}$, followed by blocking in 10\% goat serum for $1 \mathrm{~h}$ at room temperature. The sections were incubated overnight at $4^{\circ} \mathrm{C}$ with the primary rabbit polyclonal SYK antibody (1:50 dilution). Sections were then washed and incubated with FITC-conjugated AffiniPure goat antirabbit IgG (1:50 dilution) for $30 \mathrm{~min}$ at $37^{\circ} \mathrm{C}$. Following that, sections were counterstained with propidium iodide. After extensive washing, sections were mounted in Dabco (Air Products and Chemicals, Allentown, PA) and analyzed by confocal microscopy (TCS SP2, Leica Microsystems GmbH, Wetzlar, Germany).

\section{RNA Extraction and Plasmid Construction}

Total RNA was isolated from mammary tissues using TRIzol reagent, according to standard protocols. The RNA quantity and purity were determined by absorbance at $260 \mathrm{~nm}$ using a NanoDrop 2000c spectrophotometer (Thermo Fisher Scientific). Ribonucleic acid integrity was analyzed by denaturing agarose gel electrophoresis with the $28 \mathrm{~S}$ rRNA band appearing approximately twice as intense as the $18 \mathrm{~S}$ rRNA band. Total RNA was reverse transcribed using M-MLV with oligo $\mathrm{d}(\mathrm{T})$ at $37^{\circ} \mathrm{C}$ for $50 \mathrm{~min}$. The cDNA samples were stored at $-20^{\circ} \mathrm{C}$ until PCR amplification.

To construct SYK expression plasmids, full-length DNA carrying the dairy cow $S Y K$ coding region was generated by PCR. The sequences of the PCR primers used were as follows: forward $=5^{\prime}$-AACTGCAGATGGGTGACGGCGCCAACAAC-3'; reverse = 5'-TCCCCGCGGGGGAGCCTTTAGCCTTTAGT-

TAACCACGTCG-3' ${ }^{\prime}$. The PCR product was subcloned into the pIRES2-EGFP (TIANDZ, Beijing, China) and pcDNA3.1(+) (Invitrogen, Carlsbad, CA) plasmids at the PstI/SacII sites. To construct short hairpin RNA (shRNA) directed against $S Y K$ and a negative scrambled control, 4 pairs of oligonucleotides specific for the dairy cow $S Y K$ sequence and 1 pair of nontargeting oligonucleotides were designed and subcloned into pSilencer1.0-U6 (Ambion, Carlsbad, CA) at the EcoRI/ApaI sites (Table 2). These $4 S Y K$ shRNA were termed RNA interference (RNAi) 1, RNAi2, RNAi3, and RNAi4. The nontargeting shRNA plasmid was termed RNAiNC and used as a negative control.

To clone and analyze the AKT1 promoter, genomic DNA was isolated from mammary tissues using a mammalian genomic DNA extraction kit. The 5'-flanking region of dairy cow $A K T 1$ was generated by PCR. The amplified DNA fragments, $-2,000 /+83,-904 /+83$, $-439 /+83$, and $-84 /+83$ were subcloned into the luciferase reporter vector pGL3 (Promega) at the KpnI/ Hin-

Table 2. Short hairpin RNA (shRNA) used in this study ${ }^{1}$

\begin{tabular}{ll}
\hline shRNA & Sequences $\left(5^{\prime}-3^{\prime}\right)$ \\
\hline RNAi1 & F: CGGAGCTGAATGGCACCTATTTCAAGAGAATAGGTGCCATTCAGCTCCTTTTTG \\
& R: AATTCAAAAAGGAGCTGAATGGCACCTATTCTCTTGAAATAGGTGCCATTCAGCTCCGGGCC \\
RNAi2 & F:CGTCGAGCATTATTCTTATATTCAAGAGATATAAGAATAATGCTCGACTTTTTG \\
& R:AATTCAAAAATCGAGCATTATTCTTATATCTCTTGAATATAAGAATAATGCTCGACGGGCC \\
RNAi3 & F:CGTGGATAGTTCTACAACATTTCAAGAGAATGTTGTAGAACTATCCACTTTTG \\
& R:AATTCAAAAAGTGGATAGTTCTACAACATTCTCTTGAAATGTTGTAGAACTATCCACGGGCC \\
RNAi4 & F:CGCTGGTTACTCAACATTATTTCAAGAGAATAATGTTGAGTAACCAGCTTTTTG \\
RNAiNC & F:AATTCAAAAAGTGGTTACTCAACATTATTCTCTTGAAATAATGTTGAGATACCAGCGGGCC \\
& R:AATTCAAAAAACACCCTGGTGAACCGCATTCTCTTGAAATGCGGTTCACCAGGGTGTGGGCC \\
\hline
\end{tabular}

${ }^{1} \mathrm{~F}=$ forward; $\mathrm{R}=$ reverse; $\mathrm{RNAi}=\mathrm{RNA}$ interference with 4 pairs (1-4) of oligonucleotides specific for the dairy cow $S Y K$ sequence and a negative control (NC). 
dIII sites. The primers used were as follows: $-2,000\left(5^{\prime}\right)$ $=5^{\prime}$-CGGGGTACCGAAGCCCCCGACGTTCAC-3'; $-904\left(5^{\prime}\right)=5^{\prime}$-CGGGGTACCCCATTCCTGATTTGTTGCC-3'; $\quad-439\left(5^{\prime}\right)=$ 5'-CGGGGTACCGGGGACTGCCTGGAAGAGAG-3'; $-84\left(5^{\prime}\right)=$ 5'-CGGGGTACCTGGGCTCCCCTGAGGCTCTG-3'; $+83\left(3^{\prime}\right)=5^{\prime}$-CCCAAGCTTGCTGCTGTGGCTCCCGC-3'. The fidelity of each construct was confirmed by sequencing.

\section{Cell Culture}

Primary cell culturing of mammary epithelial cells from lactating dairy cows was performed as described with minor modifications (Huynh et al., 1991; Jedrzejczak and Szatkowska, 2014). Briefly, the tissue samples were minced using surgical scissors and digested for 2 $\mathrm{h}$ at $37^{\circ} \mathrm{C}$ with collagenase III. The digested tissue was filtered through a nylon mesh and washed with HBSS. Cells were pelleted at 1,000 rpm for $10 \mathrm{~min}$ and then washed twice in HBSS. The isolated cells were cultured in DMEM/F12 supplemented with 10\% FBS, $100 \mathrm{U} /$ $\mathrm{mL}$ of penicillin, and $100 \mu \mathrm{g} / \mathrm{mL}$ of streptomycin in cell culture flasks coated with $0.5 \%$ rat tail collagen and incubated in $5 \% \mathrm{CO}_{2}$ at $37^{\circ} \mathrm{C}$. The primary cell cultures were trypsinized at $\sim 80 \%$ confluence with $0.25 \%$ trypsin and $0.02 \%$ EDTA and passaged to remove fibroblasts. Pure mammary epithelial cells were obtained after 3 or 4 passages, and all experiments were conducted using cells within passage 10 .

\section{Transfection}

Mammary epithelial cells were plated in 6-well plates at $1 \times 10^{6}$ cells/well or in 96 -well plates at $1 \times 10^{3}$ cells / well and grown to $80 \%$ confluence. Transient transfection was conducted by Lipofectamine 2000 (Life Technologies), as described (Wang et al., 2014). Briefly, for $S Y K$ knockdown, cells were transfected with either 3 $\mu \mathrm{g}$ of $S Y K$-specific shRNA or non-targeting shRNA as a negative control in the 6 -well plate format $(0.5 \mu \mathrm{g}$ of shRNA in the 96 -well plate format). For the $S Y K$ overexpression study, cells were transfected with either $3 \mu \mathrm{g}$ of pIRES2-EGFP-SYK or pIRES2-EGFP (TIANDZ) as a negative control in the 6 -well plate format $(0.5 \mu \mathrm{g}$ of shRNA in the 96-well plate format). The transfection efficiency was assessed by specific protein levels in cell lysates isolated $48 \mathrm{~h}$ post-transfection.

\section{Cell Viability Assay}

Mammary epithelial cell viability was measured by MTT [3-(4,5-dimethylthiazol-2-yl)-2,5-diphenyltetra- zolium] assay (Haines et al., 2009). Briefly, mammary epithelial cells were plated on 96 -well plates at $1 \times 10^{3}$ cells/well. Twenty-four, 48, and $72 \mathrm{~h}$ after transfection, $20 \mu \mathrm{L}$ of $\mathrm{MTT}(5 \mathrm{mg} / \mathrm{mL})$ was added into each well and incubated at $37^{\circ} \mathrm{C}$ with $5 \% \mathrm{CO}_{2}$ for $4 \mathrm{~h}$. The wells were then decanted and the purple formazan crystals formed were dissolved in $150 \mu \mathrm{L}$ of dimethyl sulfoxide. The absorbance of the plate was taken at $492 \mathrm{~nm}$ in a microplate reader (GF-M2000, Shandong Gaomi Caihong Analytical Instruments Co. Ltd., Shandong, China). All experiments were performed in triplicate.

\section{EdU Proliferation Assay}

Mammary epithelial cell proliferation was measured by KeyFluor488 Click-iT EdU Imaging Kit (KeyGEN BioTECH, Nanjing, China) as described (Limsirichaikul et al., 2009). Briefly, cells were plated on coverslips in 6 -well plates at $1 \times 10^{6}$ cells/well. Forty-eight hours after transfection, $20 \mu M$ EdU was added into each well and cells were incubated for $2 \mathrm{~h}$. Cells were then washed with PBS, followed by fixation and permeabilization with PBS containing 3.7\% formaldehyde and $0.5 \%$ triton X-100 (Sigma-Aldrich) for $20 \mathrm{~min}$. After extensive washing with PBS containing 3\% BSA, cells were incubated with Click-iT reaction cocktail (Thermo Fisher Scientific) for $30 \mathrm{~min}$ at room temperature. Cells were then washed twice with PBS containing $3 \%$ BSA, followed by counterstaining with 4'6-diamidino2-phenylindole (DAPI) for $10 \mathrm{~min}$. Cells were washed 3 times with PBS and mounted in Dabco and analyzed by confocal microscopy (TCS SP2, Leica Microsystems, Wetzlar, GmbH, Germany). More than 5 fields were captured per well and the percentage of EdU-positive cells was defined as the proliferation rate. All experiments were performed in triplicate.

\section{Western Blotting}

Western blotting was performed as described (Wang et al., 2014). Mammary tissues from lactating cows and dry cows were used to detect SYK expression in different physiology stage of dairy cows. Mammary epithelial cells subjected to the transfection treatment described above were used to detect the expression and activity of AKT1, p42/44 MAPK, STAT5, and cyclin D1, which have been reported to regulate mammary epithelial cell proliferation in rodent or dairy cow mammary gland (Lin and Li, 2007; Nørgaard et al., 2008b; LaRocca et al., 2011). Briefly, mammary tissues or mammary epithelial cells were lysed using RIPA buffer. Protein concentrations were determined by the bicinchoninic acid (BCA) method. Approximately $40 \mu \mathrm{g}$ of protein 
was resolved by $12 \%$ gel electrophoresis and transferred to nitrocellulose membranes. Membranes were incubated with primary antibodies against SYK, cyclin D1, AKT1, phospho-AKT1, p42/44 MAPK, phosphop42/44 MAPK, STAT5, phospho-STAT5, $\beta$-CN, and $\beta$-actin at $4^{\circ} \mathrm{C}$ overnight. Membranes were washed 3 times for $5 \mathrm{~min}$ each in TBS/T and then incubated with HRP-conjugated secondary antibodies against rabbit or goat for $1.5 \mathrm{~h}$ at room temperature. Bound antibody was detected by chemiluminescence using SignalFire ECL.

\section{Luciferase Reporter Assay}

Mammary epithelial cells of dairy cows were cultured in 24-well plates at $1 \times 10^{4}$ cells/well and transiently transfected with $250 \mathrm{ng}$ of pGL3-CMV, pGL3, pGL3AKT1, or pcDNA3.1-SYK using Lipofectamine 2000. Luciferase activity assays were performed $48 \mathrm{~h}$ after transfection as described (Li et al., 2012). All experiments were performed in triplicate.

\section{Statistical Analysis}

Data are presented as the mean \pm SEM. For the expression levels of SYK in lactating and dry cows, data were analyzed by ANOVA (GraphPad Prism 6, La Jolla, CA) using the mixed-effects model with day of sampling as a fixed effect and cows as a random effect. In analyzing the effect SYK on cell viability, 2-way ANOVA with repeated measures were used. Bonferroni's multiple comparison test was used for post ANOVA comparisons. In analyzing the effect of SYK on cell proliferation, cyclin D1 expression, and signaling molecules expression involved in cell proliferation, a 2-tailed unpaired $t$-test was used for compare means between SYK knockdown or overexpression group and control group. $P<0.05$ was considered statistically significant.

\section{RESULTS}

\section{Expression of SYK in Mammary Glands of Dairy Cows}

To compare SYK expression in mammary glands of dry cows versus lactating cows, we collected mammary tissues from dry and lactating cows and performed Western blot analysis. The expression of SYK was markedly higher in dry period mammary tissues than in lactating tissues $(P<0.01$; Figure $1 \mathrm{~A}$ and $\mathrm{B})$. Immunofluorescence staining of sections of mammary tissues showed that, in the dry period mammary gland, SYK was highly enriched in the cytoplasm of ductal epithelial cells of dairy cows. The intense staining was observed on the side of the ductal lumen, indicating targeted polarization of SYK in the dry period mammary gland. In lactating tissue, SYK was diffusely localized within the cytoplasm of acinar epithelial cells, surrounding the nuclei (Figure 1C). These results suggested that SYK
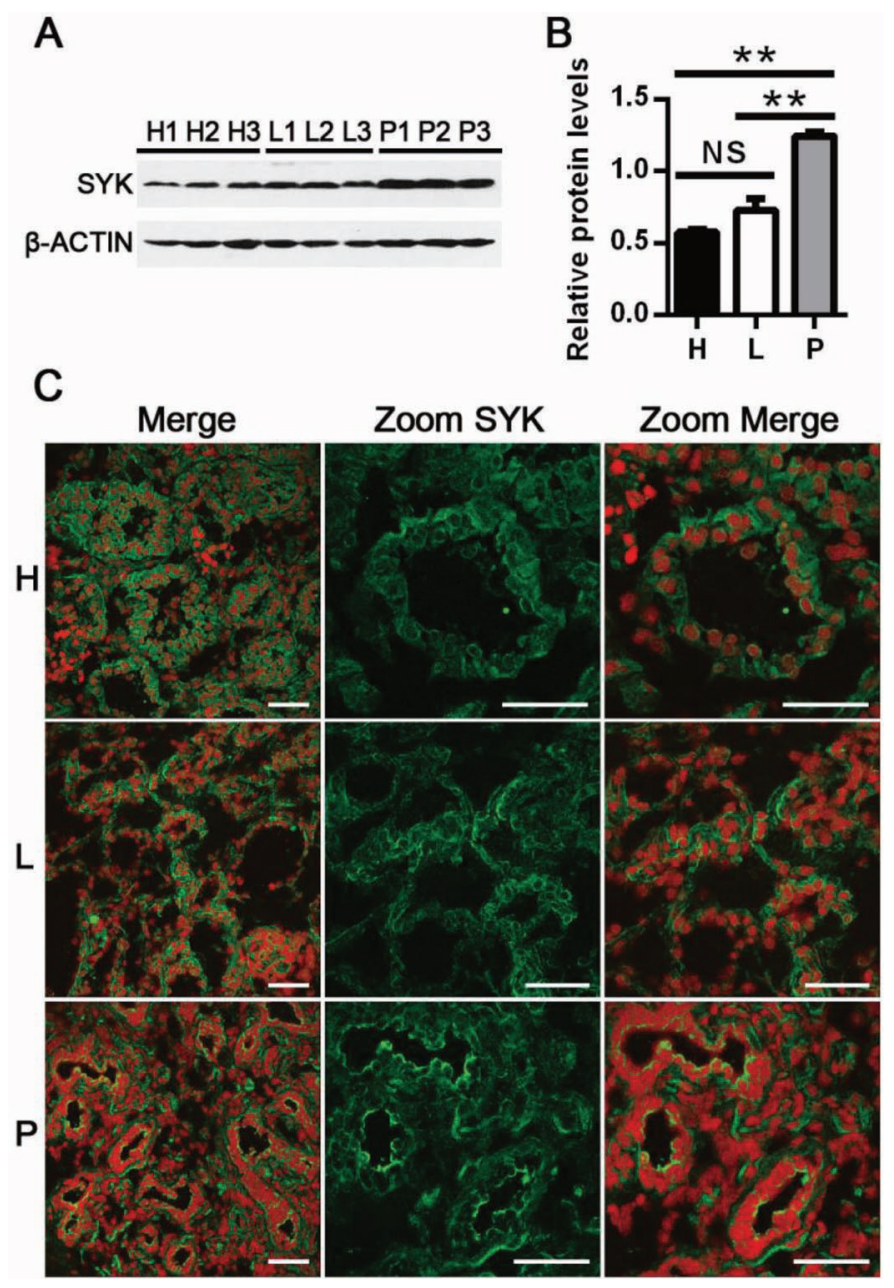

Figure 1. The expression of spleen tyrosine kinase (SYK) in mammary tissues of dairy cows. (A) Western blot analyses showing the levels of SYK in dairy cow mammary tissues. These data were obtained from tissue lysates of dairy cow mammary glands; $\beta$-actin was used as a loading control. (B) Quantification of SYK expression from the Western blots in panel A. Bars depict the signal intensity for SYK from the 3 types of mammary tissues of dairy cows. Results are shown as the mean \pm SEM. ${ }^{* *} P<0.01 ; \mathrm{NS}=$ no statistical significance. (C) Immunofluorescence staining showing the localization of SYK expressed in mammary tissues of dairy cows. The SYK was labeled by fluorescein isothiocyanate (green, light gray in grayscale version of figure), and nuclei were stained with propidium iodide (red, dark gray in grayscale version of figure). Scale bar $=50 \mu \mathrm{m} ; \mathrm{H}=$ lactating mammary tissues with high-quality milk; $\mathrm{L}=$ lactating mammary tissues with low-quality milk; $\mathrm{P}=$ mammary tissues in the dry period; Merge = representative images of SYK (green) and nuclei (red) localization; Zoom SYK = enlarged area in the relevant images; Zoom Merge = representative enlarged images of SYK and nuclei localization. Color version available online. 
has roles in mammary gland remodeling during the dry period.

To compare SYK expression in lactating cows with different milk quality, we detected SYK expression in lactating mammary tissues with high- and low-quality milk. The expression level and localization of SYK showed the similar pattern in lactating tissues with different quality milk $(P>0.05$; Figure 1A, B and $C)$. During lactation, all lactating cows were fed the same ration to requirements. The similar pattern of SYK expression in lactating cows with different quality milk suggested that SYK did not regulate metabolic processes, but had a role in maintaining cell numbers during the lactation period.

\section{Effect of SYK on Mammary Epithelial Cell Proliferation in Dairy Cows}

To investigate whether SYK affects mammary gland remodeling and lactation by mediating cell proliferation, mammary epithelial cells of dairy cows were treated with either $S Y K$-specific shRNA or nontargeting shRNA as a negative control. As shown in Figure 2A and B, $S Y K$ shRNA treatment resulted in a decrease in SYK protein expression $48 \mathrm{~h}$ after transfection. Compared with the negative control, SYK protein levels were significantly decreased in those cells that were transfected with RNAi3 or RNAi4 $(P<0.01)$. Therefore, RNAi3 was chosen for subsequent SYK knockdown experiments to examine the effect of SYK knockdown on mammary epithelial cell proliferation. The MTT results showed that, compared with the control, SYK knockdown significantly decreased the viability of mammary epithelial cells 48 and $72 \mathrm{~h}$ after transfection $(P<0.05)$, but not $24 \mathrm{~h}$ after transfection (Figure $2 \mathrm{C}$ ). Then we assessed the effects of SYK knockdown on mammary epithelial cell proliferation $48 \mathrm{~h}$ after transfection. As shown in Figure 2D and 2E, compared with the control SYK knockdown significantly decreased EdU incorporation in mammary epithelial cells of dairy cows $(P<0.05)$. Meanwhile, the expression of cyclin D1, which is a regulator of the cell cycle, was also significantly decreased in SYK knockdown cells $(P<0.05$; Figure $2 \mathrm{~F}$ and $\mathrm{G})$.

Next, we examined the effect of SYK overexpression on mammary epithelial cell proliferation. Compared with cells carrying the empty pIRES2-EGFP vector, the levels of SYK in dairy cow mammary epithelial cells were significantly increased by transfection with pIRES2-EGFP-SYK vector $(P<0.01$; Figure $3 \mathrm{~A}$ and B). Meanwhile, the overexpression of SYK in dairy cow mammary epithelial cells markedly enhanced cell viability 48 and $72 \mathrm{~h}$ after transfection $(P<0.01$; Figure $3 \mathrm{C})$, as well as cell proliferation $(P<0.01$; Figure 3D and $\mathrm{E})$ and cyclin D1 expression $(P<0.01$; Figure $3 \mathrm{~F}$
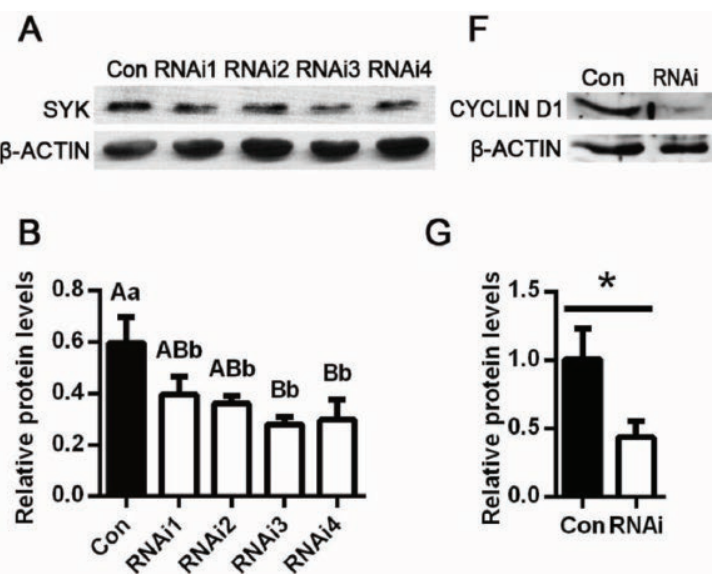

G

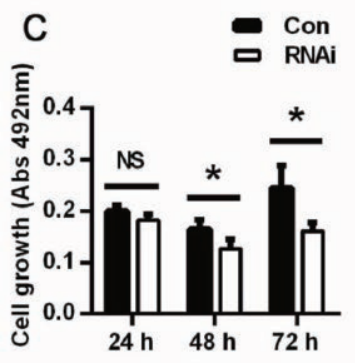

E
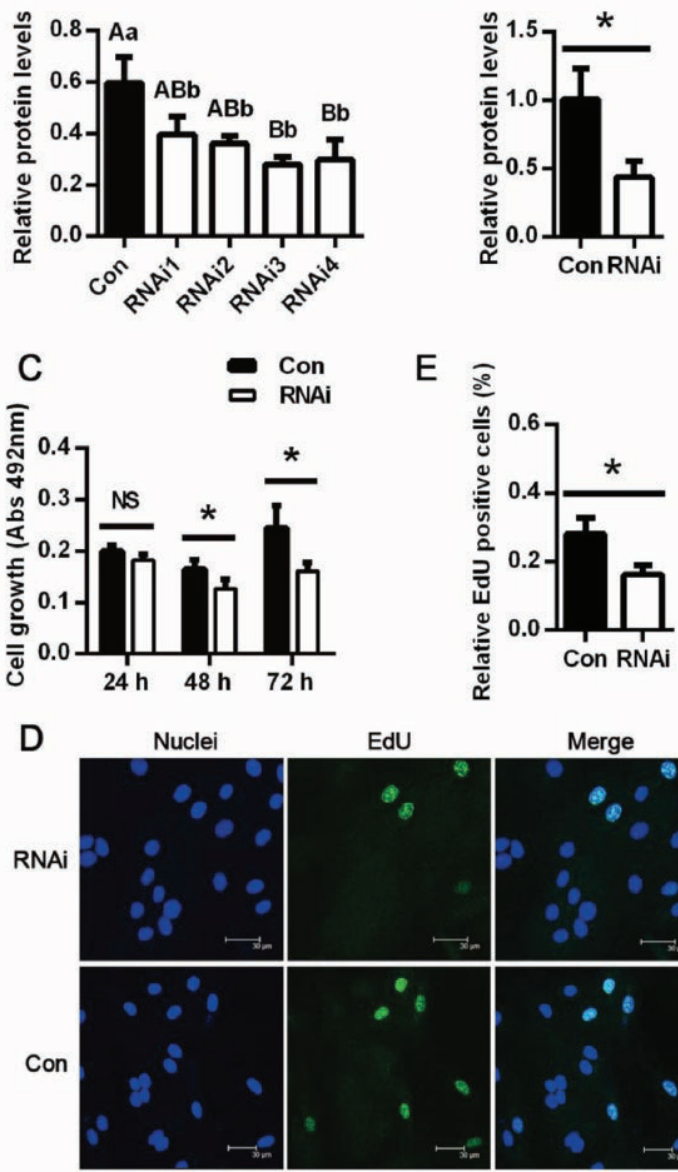

Figure 2. The effect of spleen tyrosine kinase (SYK) knockdown on mammary epithelial cell proliferation. (A) Western blot analyses show the levels of SYK and $\beta$-actin (loading control) in SYK knockdown and RNA interference (RNAi) control (Con) mammary epithelial cells. (B) Quantification of SYK expression from the Western blots in panel A. Bars depict the signal intensity for SYK from mammary epithelial cells. (C) Mammary epithelial cell viability in SYK knockdown cells 24, 48, and $72 \mathrm{~h}$ after transfection. (D) Mammary epithelial cell proliferation was assessed by 5-ethynyl-2'-deoxyuridine (EdU) incorporation in SYK knockdown cells $48 \mathrm{~h}$ after transfection. The DAPI (4',6-diamidino-2-phenylindole)-stained nuclei are blue (dark gray in grayscale version of figure) and EdU-labeled nuclei are green (light gray in grayscale version of figure). Merge images show the overlap between nuclei and regions of EdU incorporation. Scale bar $=30 \mu \mathrm{m}$.

(E) The percentage of EdU-positive cells out of the total cells in the culture. (F) Western blot analyses show the levels of cyclin D1 and $\beta$-actin (loading control) in SYK knockdown and RNAi control (Con) mammary epithelial cells. (G) Quantification of cyclin D1 expression from the Western blots in panel F. Bars depict the signal intensity for cyclin D1 from mammary epithelial cells. Results are shown as the mean \pm SEM from 3 independent experiments, each of which was performed in triplicate. For panel B, means without a common lowercase letter $(\mathrm{a}, \mathrm{b})$ differ at $P<0.05$, and means without a common uppercase letter $(\mathrm{A}, \mathrm{B})$ differ at $P<0.01 .{ }^{*} P<0.05$; ${ }^{* *} P<0.01$; NS $=$ no statistical significance. Color version available online. 

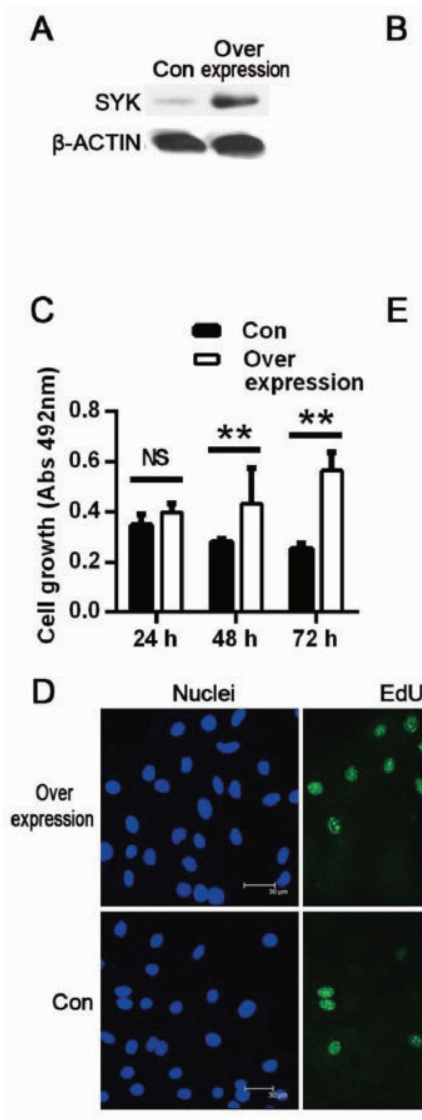

$\mathbf{F}$

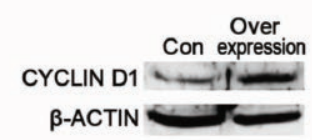

B
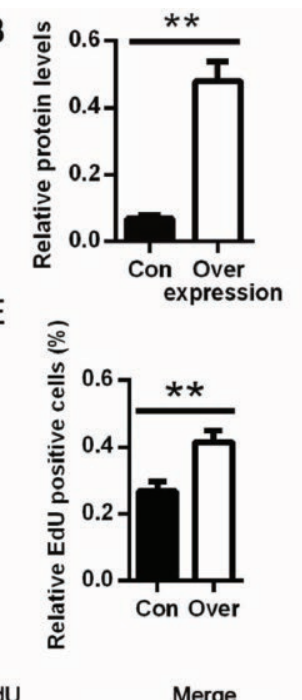

EdU

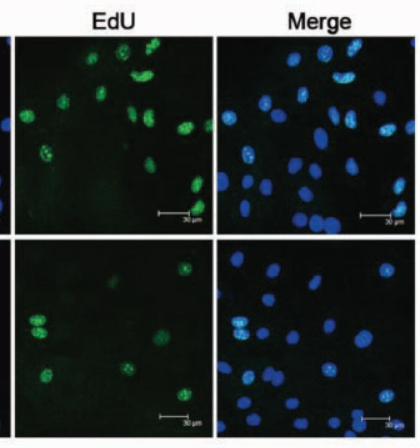

G

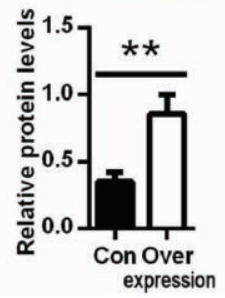

Figure 3. The effect of spleen tyrosine kinase (SYK) overexpression on mammary epithelial cell proliferation. (A) Western blot analyses show the levels of SYK and $\beta$-actin (loading control) in SYKoverexpressed mammary epithelial cells. (B) Quantification of SYK expression from the Western blots in panel A. Bars depict the signal intensity for SYK from mammary epithelial cells. (C) Mammary epithelial cell viability in SYK-overexpressed cells 24,48 , and $72 \mathrm{~h}$ after transfection. (D) Mammary epithelial cell proliferation was assessed by 5-ethynyl-2'-deoxyuridine (EdU) incorporation in SYKoverexpressed cells $48 \mathrm{~h}$ after transfection. The DAPI (4',6-diamidino2 -phenylindole)-stained nuclei are blue (dark gray in grayscale version of figure) and EdU-labeled nuclei are green (light gray in grayscale version of figure). Merge images show the overlap between nuclei and regions of EdU incorporation. Scale bar $=30 \mu \mathrm{m}$. (E) The percentage of EdU-positive cells out of the total cells in the culture. (F) Western blot analyses show the levels of cyclin D1 and $\beta$-actin (loading control) in SYK-overexpressed mammary epithelial cells. (G) Quantification of cyclin D1 expression from the Western blots in panel F. Bars depict the signal intensity for cyclin D1 from mammary epithelial cells. In the control group (Con), cells were transfected with empty pIRES2EGFP vector. In the overexpression group, cells were transfected with pIRES2-EGFP-SYK vector. Results are shown as the mean \pm SEM from 3 independent experiments, each of which was performed in triplicate. ${ }^{*} P<0.05 ;{ }^{*} P<0.01 ; \mathrm{NS}=$ no statistical significance. Color version available online.

and G). Overall, these data strongly support the notion that SYK is crucial in mediating mammary epithelial cell proliferation in mammary glands of dairy cows.

To further explore the regulatory pathway by which SYK mediates mammary epithelial cell proliferation, we examined the levels of AKT1, phospho-AKT1, p42/44 MAPK, phospho-p42/44 MAPK, STAT5, and phospho-STAT5, which mediate cell survival and proliferation (Garrington and Johnson, 1999; Ackler et al., 2002). Compared with the control, the protein levels of AKT1, phospho-AKT1, p42/44 MAPK $(P<$ 0.05), phospho-p42/44 MAPK, and STAT5 $(P<0.01)$ were markedly reduced in SYK knockdown mammary epithelial cells $48 \mathrm{~h}$ after transfection, whereas levels of phospho-STAT5 remained relatively unchanged (Figure 4A and C; Supplemental Figure S1A, http://dx.doi. org/10.3168/jds.2015-10118). In contrast, the overexpression of SYK in dairy cow mammary epithelial cells markedly increased the levels of AKT1, phosphoAKT1, p42/44 MAPK $(P<0.05)$, phospho-p42/44 MAPK $(P<0.01)$, and STAT5 $(P<0.05)$, but not phospho-STAT5 (Figure 4B and D; Supplemental Figure S1B). To determine whether SYK also affects mammary epithelial cell secretory activity, we examined the expression of $\beta$-CN in SYK knockdown cells and in cells overexpressing SYK. We found that SYK knockdown significantly decreased $\beta$-CN expression $(P<0.05)$. In contrast, overexpression of SYK significantly enhanced $\beta$-CN expression $(P<0.01)$. Taken together, these results are consistent with the hypothesis that SYK mediates mammary epithelial cell proliferation in mammary glands of dairy cows.

\section{SYK Mediates Mammary Epithelial Cell Proliferation by Regulating AKT1 Transcription}

As AKT1 expression and activity are strongly activated by SYK in mammary epithelial cells of dairy cows, we examined whether AKT1 is a mediator of SYK-controlled mammary epithelial cell proliferation. We generated luciferase reporters fused to the promoter region of $A K T 1$. To investigate the activator sites for the induction of AKT1 expression in mammary epithelial cells of dairy cows, the luciferase assay was performed with clones containing various deletions in the AKT1 promoter. Deletion to position -904 and -439 bp resulted in progressively higher promoter activity. In contrast, lower activity was apparent with the region containing -84 to $+83 \mathrm{bp}$. These data indicated that the region from -439 to $-84 \mathrm{bp}$ contains positive ciselements (Figure 5A).

We then examined whether the AKT1 promoter could be induced by SYK. A luciferase reporter assay was performed with mammary epithelial cells transfected 


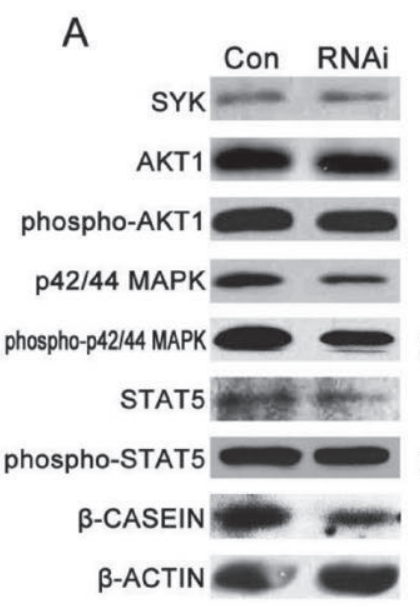

B

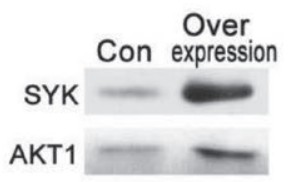

phospho-AKT1

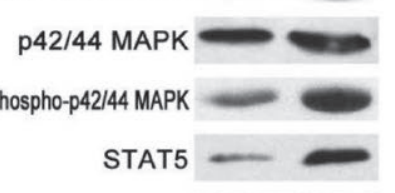

phospho-STAT5

B-CASEIN

$\beta$-ACTIN

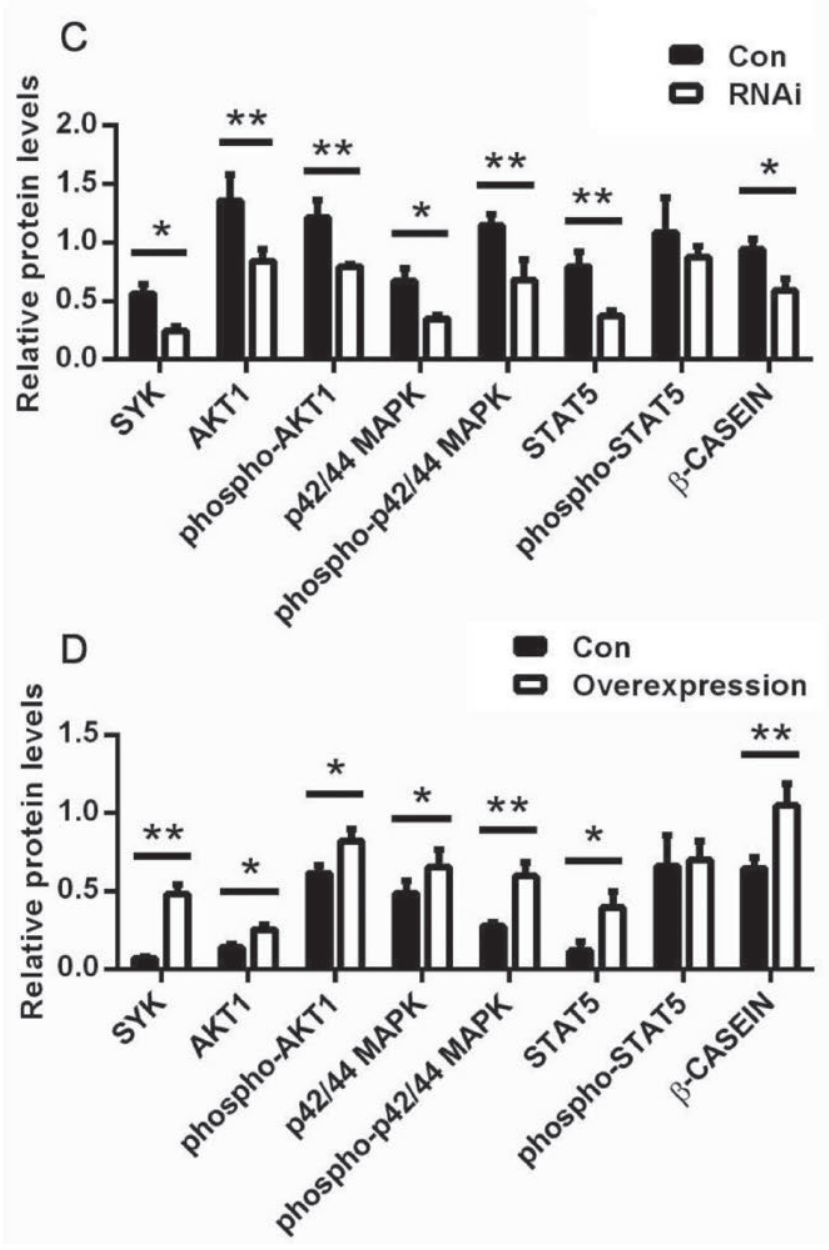

Figure 4. Spleen tyrosine kinase (SYK) mediates mammary epithelial cell proliferation by regulating AKT1, MAPK, and STAT5 levels. (A) Western blot analyses show the levels of $\beta$-CN and signal molecules involved in cell proliferation in SYK-knockdown mammary epithelial cells. (B) Western blot analyses show the levels of $\beta-\mathrm{CN}$ and signal molecules involved in cell proliferation in mammary epithelial cells that overexpress SYK. (C) Quantification of protein levels from the Western blots in panel A. (D) Quantification of protein levels from the Western blots in panel B. For C and D, results are shown as the mean \pm SEM from 3 independent experiments, each of which was performed in triplicate. ${ }^{*} P<0.05 ;{ }^{*} P<0.01$. with pGL3-AKT1 $(-439 /+83)$ and pcDNA3.1-SYK. As shown in Figure 5B, compared with the control, expression of pGL3-AKT1 $(-439 /+83)$ alone induced the reporter activity, whereas co-expression of pGL3AKT1 $(-439 /+83)$ and pcDNA3.1-SYK significantly stimulated the AKT1 promoter activity. These results provide evidence that the promoter activity of $A K T 1$ is enhanced by SYK in mammary epithelial cells of dairy cows.

\section{DISCUSSION}

Spleen tyrosine kinase is implicated in breast cancer progression and invasion (Coopman et al., 2000); however, little is known about its function in normal mammary gland. Expression of SYK has been demonstrated in mammary epithelial cells of mice and in the human mammary epithelial cell line MCF-10A (Ruschel and Ullrich, 2004; Neuhaus et al., 2011). In this study, we found that SYK was expressed in normal mammary glands of dairy cows. The levels of SYK were markedly

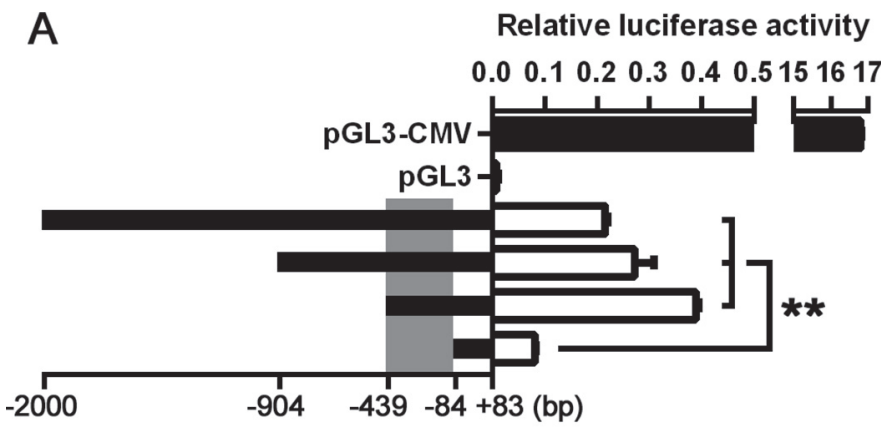

B

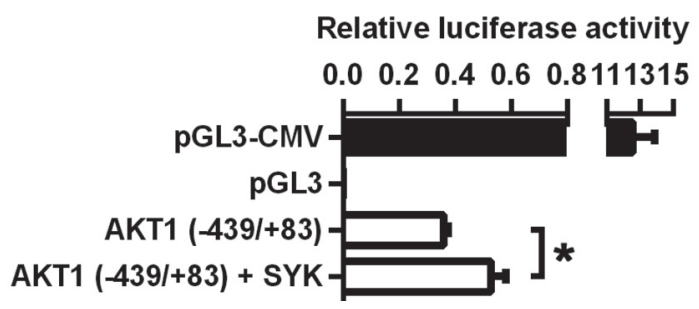

Figure 5. Spleen tyrosine kinase (SYK) activates the AKT1 promoter. (A) Effect of deletions on $A K T 1$ promoter activity in mammary epithelial cells of dairy cows. Four deletion mutants of the AKT1 promoter were transfected into mammary epithelial cells of dairy cows, and luciferase activity was measured $48 \mathrm{~h}$ after transfection. The gray box indicates the region from -439 to $-84 \mathrm{bp}$. (B) SYK induces AKT1 promoter $(-439 /+83)$ luciferase activity. Mammary epithelial cells of dairy cows were transfected with pGL3-AKT1 $(-439 /+83)$ or were co-transfected with pGL3-AKT1 $(-439 /+83)$ and pcDNA3.1-SYK. Luciferase activity was measured $48 \mathrm{~h}$ after transfection. In the positive control group, cells were transfected with pGL3-CMV vector. In the negative control group, cells were transfected with pGL3 vector. Results are shown as the mean \pm SEM from 3 independent experiments, each of which was performed in triplicate. ${ }^{*} P<0.05$; ${ }^{*} P<$ 0.01 . 
higher in dry period mammary tissues than in lactating mammary tissues, whereas the levels of SYK were similar in lactating tissues with high- and low-quality milk. As all lactating cows were fed the same ration to requirements, the similar pattern of SYK expression in the 2 lactating groups indicated that SYK was not a metabolic synthesis controller. In B cells, SYK is capable of regulating development and proliferation (Uckun et al., 2010). Here, we provide the first evidence that SYK is able to induce mammary epithelial cell proliferation.

We examined EdU incorporation in mammary epithelial cells of dairy cows when SYK was knocked down or overexpressed, and found that a proliferation effect was observed after $48 \mathrm{~h}$ of treatment. The pattern of SYK expression in lactating and dry mammary glands and the function of SYK in inducing proliferation suggested that SYK has a role in maintaining lactation and remodeling (in dry period mammary glands) in dairy cows. During the dry period between successive lactations, the mammary gland of dairy cows undergoes extensive remodeling that is marked by phases of involution and mammogenesis (De Vries et al., 2010). Cell proliferation is the major determinant of cell turnover at this stage (Nørgaard et al., 2008b). Western blot and immunofluorescence results showed that SYK was highly enriched in the dry period mammary gland and mainly located in ductal epithelial cells, indicating that ductal epithelial cell proliferation is occurring, which is consistent with a report that during this time the luminal area in mammary tissue increases (Hurley, 1989). In contrast, during lactation, milk production is likely a function of the number and secretory activity of epithelial cells in ruminants (Knight and Peaker, 1984; Capuco et al., 2003). Western blot and immunofluorescence results showed that in lactating tissue, SYK expression was lower and specifically located in acinar epithelial cells. These data are in agreement with a report that, during lactation, mammary cell proliferation is low (Sorensen et al., 2006), with only $0.3 \%$ of mammary epithelial cells proliferating in a 24 -h period (Capuco et al., 2001). Yet this proliferative rate is sufficient to replace most mammary epithelial cells by the end of lactation.

In mammary epithelial cells, multiple signaling cascades are involved in cell proliferation. The MAPK are associated with cell proliferation in response to growth factor stimulation (Fata et al., 2007; Lin and Li, 2007). In the present study, we found that the nonreceptor tyrosine kinase SYK induced and activated p42/44 MAPK in mammary epithelial cells of dairy cows, supporting the role of SYK in stimulation of mammary epithelial cell proliferation in dairy cows.
In rodents, STAT5 is essential for alveologenesis (Liu et al., 1997). Loss of STAT5 before pregnancy prevents epithelial cell proliferation and differentiation (Miyoshi et al., 2001; Cui et al., 2004), whereas overexpression of hyperactive STAT5 in mouse mammary epithelium causes a high degree of acinar epithelial cell proliferation (Iavnilovitch et al., 2002). In cows, STAT5 mRNA significantly increased at -16 and $4 \mathrm{~d}$ from parturition (Nørgaard et al., 2008b). Both DNA-binding activity and phosphorylation of STAT5 in bovine mammary gland are at near maximal levels in late pregnancy, which points to a role in regulation of mammary cell turnover (Wheeler et al., 2001). In this study, we observed that SYK expression was higher in dry cows and had the ability to induce STAT5 expression in mammary epithelial cells of dairy cows. Under physiological conditions, cytoplasmic STAT are phosphorylated on tyrosine residues by nonreceptor tyrosine kinases of the Janus and Src families for cytokine receptor activation (Darnell et al., 1994; Calò et al., 2003). We did not, however, note a change in STAT5 phosphorylation in response to changes in SYK expression in cultured mammary epithelial cells of dairy cows.

The serine and threonine protein kinase, ATK1, is activated by a variety of signals via PI3K-dependent mechanisms (Feng et al., 2004); AKT1 also has a key role in cell survival pathways by inducing cell proliferation and inhibiting the apoptosis process. In $A K T 1^{-/-}$ mice, the epithelial component of mammary gland is underdeveloped relative to wild-type mice (Maroulakou et al., 2008). In mouse fibroblast NIH3T3 cells, the nonreceptor tyrosine kinase Src significantly induces AKT1 promoter activity (Park et al., 2005). In the present study, we found that SYK has the ability to induce AKT1 expression and activation in mammary epithelial cells of dairy cows, implying that AKT1 may be regulated at the translational and post-translational levels by SYK in mammary gland. In this study, we cloned the AKT1 promoter of dairy cow and demonstrated the region from -439 to -84 bp contained positive cis-elements essential for $A K T 1$ expression under normal conditions. The luciferase assay with the region from -439 to $-84 \mathrm{bp}$ indicates that the promoter of dairy cow $A K T 1$ gene occurs in response to SYK expression, suggesting that SYK also regulates $A K T 1$ at the transcriptional level in mammary epithelial cells of dairy cows.

In conclusion, SYK is expressed in dry period and lactating mammary glands of dairy cows and regulates mammary epithelial cell proliferation in mammary gland lactation cycle. Cell proliferation is regulated by SYK by inducing AKT1, p42/44 MAPK, and STAT5 expression. Furthermore, we present evidence that SYK 
regulates AKT1 expression via promoter activation. Expression of $A K T 1$ is induced by SYK through interacting with the promoter region between -439 and -84 bp in mammary epithelial cells of dairy cows.

\section{ACKNOWLEDGMENTS}

This work was supported by grants from the National Natural Science Foundation of China (31200984), the Heilongjiang Province Postdoctoral Funded Project (LBH-Z14031, China) and Northeast Agricultural University Research Foundation for Scientific Talents (2010RCB49, China). All authors read and approved the final manuscript and have declared that no competing interests exist.

\section{REFERENCES}

Ackler, S., S. Ahmad, C. Tobias, M. D. Johnson, and R. I. Glazer. 2002. Delayed mammary gland involution in MMTV-AKT1 transgenic mice. Oncogene 21:198-206.

Calò, V., M. Migliavacca, V. Bazan, M. Macaluso, M. Buscemi, N. Gebbia, and A. Russo. 2003. STAT proteins: From normal control of cellular events to tumorigenesis. J. Cell. Physiol. 197:157-168.

Capuco, A. V., R. M. Akers, and J. J. Smith. 1997. Mammary growth in Holstein cows during the dry period: Quantification of nucleic acids and histology. J. Dairy Sci. 80:477-487.

Capuco, A. V., S. E. Ellis, S. A. Hale, E. Long, R. A. Erdman, X. Zhao, and M. J. Paape. 2003. Lactation persistency: Insights from mammary cell proliferation studies. J. Anim. Sci. 81(Suppl 3):18-31.

Capuco, A. V., D. L. Wood, R. Baldwin, K. Mcleod, and M. J. Paape. 2001. Mammary cell number, proliferation, and apoptosis during a bovine lactation: Relation to milk production and effect of bST. J. Dairy Sci. 84:2177-2187.

Coopman, P. J., M. T. Do, M. Barth, E. T. Bowden, A. J. Hayes, E. Basyuk, J. K. Blancato, P. R. Vezza, S. W. McLeskey, P. H. Mangeat, and S. C. Mueller. 2000. The Syk tyrosine kinase suppresses malignant growth of human breast cancer cells. Nature 406:742-747.

Cui, Y., G. Riedlinger, K. Miyoshi, W. Tang, C. Li, C. X. Deng, G. W. Robinson, and L. Hennighausen. 2004. Inactivation of Stat5 in mouse mammary epithelium during pregnancy reveals distinct functions in cell proliferation, survival, and differentiation. Mol. Cell. Biol. 24:8037-8047.

Darnell, J. E. Jr., I. M. Kerr, and G. R. Stark. 1994. Jak-STAT pathways and transcriptional activation in response to IFNs and other extracellular signaling proteins. Science 264:1415-1421.

De Vries, L. D., H. Dover, T. Casey, M. J. VandeHaar, and K. Plaut. 2010. Characterization of mammary stromal remodeling during the dry period. J. Dairy Sci. 93:2433-2443.

Dessauge, F., V. Lollivier, B. Ponchon, R. Bruckmaier, L. Finot, S. Wiart, E. Cutullic, C. Disenhaus, S. Barbey, and M. Boutinaud. 2011. Effects of nutrient restriction on mammary cell turnover and mammary gland remodeling in lactating dairy cows. J. Dairy Sci. 94:4623-4635.

Fata, J. E., H. Mori, A. J. Ewald, H. Zhang, E. Yao, Z. Werb, and M. J. Bissell. 2007. The MAPK(ERK-1,2) pathway integrates distinct and antagonistic signals from TGFalpha and FGF7 in morphogenesis of mouse mammary epithelium. Dev. Biol. 306:193-207.

Feng, J., J. Park, P. Cron, D. Hess, and B. A. Hemmings. 2004. Identification of a PKB/Akt hydrophobic motif Ser-473 kinase as DNAdependent protein kinase. J. Biol. Chem. 279:41189-41196.

Flück, M., G. Zürcher, A. C. Andres, and A. Ziemiecki. 1995. Molecular characterization of the murine syk protein tyrosine kinase cDNA, transcripts and protein. Biochem. Biophys. Res. Commun. 213:273-281.
Garrington, T. P., and G. L. Johnson. 1999. Organization and regulation of mitogen-activated protein kinase signaling pathways. Curr. Opin. Cell Biol. 11:211-218.

Haines, E., P. Minoo, Z. Feng, N. Resalatpanah, X. M. Nie, M. Campiglio, L. Alvarez, E. Cocolakis, M. Ridha, M. Di Fulvio, J. GomezCambronero, J. J. Lebrun, and S. Ali. 2009. Tyrosine phosphorylation of Grb2: Role in prolactin/epidermal growth factor cross talk in mammary epithelial cell growth and differentiation. Mol. Cell. Biol. 29:2505-2520.

Haug, A., A. T. Høstmark, and O. M. Harstad. 2007. Bovine milk in human nutrition-A review. Lipids Health Dis. 6:25.

Hurley, W. L. 1989. Mammary gland function during involution. J. Dairy Sci. 72:1637-1646.

Huynh, H. T., G. Robitaille, and J. D. Turner. 1991. Establishment of bovine mammary epithelial cells (MAC-T): an in vitro model for bovine lactation. Exp. Cell Res. 197:191-199.

Iavnilovitch, E., B. Groner, and I. Barash. 2002. Overexpression and forced activation of stat5 in mammary gland of transgenic mice promotes cellular proliferation, enhances differentiation, and delays postlactational apoptosis. Mol. Cancer Res. 1:32-47.

Jedrzejczak, M., and I. Szatkowska. 2014. Bovine mammary epithelial cell cultures for the study of mammary gland functions. In Vitro Cell. Dev. Biol. Anim. 50:389-398.

Jiang, K., B. Zhong, C. Ritchey, D. L. Gilvary, E. Hong-Geller, S. Wei, and J. Y. Djeu. 2003. Regulation of Akt-dependent cell survival by Syk and Rac. Blood 101:236-244.

Kanie, T., A. Abe, T. Matsuda, Y. Kuno, M. Towatari, T. Yamamoto, H. Saito, N. Emi, and T. Naoe. 2004. TEL-Syk fusion constitutively activates PI3-K/Akt, MAPK and JAK2-independent STAT5 signal pathways. Leukemia 18:548-555.

Knight, C. H., and M. Peaker. 1984. Mammary development and regression during lactation in goats in relation to milk secretion. Q. J. Exp. Physiol. 69:331-338.

Kulathu, Y., G. Grothe, and M. Reth. 2009. Autoinhibiton and adapter function of syk. Immunol. Rev. 232:286-299.

LaRocca, J., J. Pietruska, and M. Hixon. 2011. Akt1 is essential for postnatal mammary gland development, function, and the expression of Btn1a1. PLoS ONE 6:e24432.

Li, H. M., C. M. Wang, Q. Z. Li, and X. J. Gao. 2012. MiR-15a decreases bovine mammary epithelial cell viability and lactation and regulates growth hormone receptor expression. Molecules 17:12037-12048.

Limsirichaikul, S., A. Niimi, H. Fawcett, A. Lehmann, S. Yamashita, and T. Ogi. 2009. A rapid non-radioactive technique for measurement of repair synthesis in primary human fibroblasts by incorporation of ethynyl deoxyuridine (EdU). Nucleic Acids Res. 37:e31.

Lin, Y., and Q. Li. 2007. Expression and function of leptin and its receptor in mouse mammary gland. Sci. China C Life Sci. 50:669675.

Liu, X., G. W. Robinson, K. U. Wagner, L. Garrett, A. WynshawBoris, and L. Hennighausen. 1997. Stat5a is mandatory for adult mammary gland development and lactogenesis. Genes Dev. 11:179-186.

Maroulakou, I. G., W. Oemler, S. P. Naber, I. Klebba, C. Kuperwasser, and P. N. Tsichlis. 2008. Distinct roles of the three Akt isoforms in lactogenic differentiation and involution. J. Cell. Physiol. 217:468-477.

Miyoshi, K., J. M. Shillingford, G. H. Smith, S. L. Grimm, K. U. Wagner, T. Oka, J. M. Rosen, G. W. Robinson, and L. Hennighausen. 2001. Signal transducer and activator of transcription (Stat) 5 controls the proliferation and differentiation of mammary alveolar epithelium. J. Cell Biol. 155:531-542.

Neuhaus, B., S. Bühren, B. Böck, F. Alves, W. F. Vogel, and F. Kiefer. 2011. Migration inhibition of mammary epithelial cells by Syk is blocked in the presence of DDR1 receptors. Cell. Mol. Life Sci. 68:3757-3770.

Nissen, A., E. Bendixen, K. L. Ingvartsen, and C. M. Røntved. 2013. Expanding the bovine milk proteome through extensive fractionation. J. Dairy Sci. 96:7854-7866.

Nørgaard, J. V., M. T. Sørensen, P. K. Theil, J. Sehested, and K. Sejrsen. 2008a. Effect of pregnancy and feeding level on cell turnover 
and expression of related genes in the mammary tissue of lactating dairy cows. Animal 2:588-594.

Nørgaard, J. V., P. K. Theil, M. T. Sørensen, and K. Sejrsen. 2008b. Cellular mechanisms in regulating mammary cell turnover during lactation and dry period in dairy cows. J. Dairy Sci. 91:2319-2327.

Ollier, S., C. Robert-Granié, L. Bernard, Y. Chilliard, and C. Leroux. 2007. Mammary transcriptome analysis of food-deprived lactating goats highlights genes involved in milk secretion and programmed cell death. J. Nutr. 137:560-567.

Park, S., D. Kim, S. Kaneko, K. M. Szewczyk, S. V. Nicosia, H. Yu, R. Jove, and J. Q. Cheng. 2005. Molecular cloning and characterization of the human AKT1 promoter uncovers its up-regulation by the Src/Stat3 pathway. J. Biol. Chem. 280:38932-38941.

Ruschel, A., and A. Ullrich. 2004. Protein tyrosine kinase Syk modulates EGFR signalling in human mammary epithelial cells. Cell. Signal. 16:1249-1261.

Seeth, Mt., M. Hoedemaker, and V. Krömker. 2015. Physiological processes in the mammary gland tissue of dairy cows during the dry period. Berl. Munch. Tierarztl. Wochenschr. 128:76-83.

Singh, K. A. J. Molenaar, K. M. Swanson, B. Gudex, J. A. Arias, R. A. Erdman, and K. Stelwagen. 2012. Epigenetics: A possible role in acute and transgenerational regulation of dairy cow milk production. Animal 6:375-381.
Sorensen, M. T., J. V. Nørgaard, P. K. Theil, M. Vestergaard, and K. Sejrsen. 2006. Cell turnover and activity in mammary tissue during lactation and the dry period in dairy cows. J. Dairy Sci. 89:4632-4639.

Stefanon, B., M. Colitti, G. Gabai, C. H. Knight, and C. J. Wilde. 2002. Mammary apoptosis and lactation persistency in dairy animals. J. Dairy Res. 69:37-52.

Uckun, F. M., S. Qazi, H. Ma, L. Tuel-Ahlgren, and Z. Ozer. 2010. STAT3 is a substrate of SYK tyrosine kinase in B-lineage leukemia/lymphoma cells exposed to oxidative stress. Proc. Natl. Acad. Sci. USA 107:2902-2907.

Wang, L., Y. Lin, Y. Bian, L. Liu, L. Shao, L. Lin, B. Qu, F. Zhao, X. Gao, and Q. Li. 2014. Leucyl-tRNA synthetase regulates lactation and cell proliferation via mTOR signaling in dairy cow mammary epithelial cells. Int. J. Mol. Sci. 15:5952-5969.

Wang, M., S. Moisá, M. J. Khan, J. Wang, D. Bu, and J. J. Loor. 2012. MicroRNA expression patterns in the bovine mammary gland are affected by stage of lactation. J. Dairy Sci. 95:6529-6535.

Wheeler, T. T., M. K. Broadhurst, H. B. Sadowski, V. C. Farr, and C. G. Prosser. 2001. Stat5 phosphorylation status and DNA-binding activity in the bovine and murine mammary glands. Mol. Cell. Endocrinol. 176:39-48. 